\title{
AN EXAMPLE OF HILTON AND ROITBERG
}

\author{
ALLAN J. SIERADSKI
}

Abstract. In [3], P. J. Hilton and J. Roitberg illustrated with several examples the failure of cancellation for products in the homotopy category of finite $\mathrm{CW}$ complexes. We reconstruct here these examples from a different point of view.

1. Introduction. Each example of Hilton and Roitberg consisted of principal $S^{3}$-bundles $E_{\alpha}$ and $E_{\beta}$ over $S^{n}$ for which $E_{\alpha} \times S^{3} \simeq E_{\beta} \times S^{3}$ and yet $E_{\alpha} \not \chi E_{\beta}$. If $B_{S^{3}}$ is the classifying space of the Lie group $S^{3}$ and if $\alpha \in \pi_{n-1}\left(S^{3}\right)$ and $\alpha_{0} \in \pi_{n}\left(B_{S^{3}}\right)$ correspond under the canonical isomorphism, then denote by $p_{\alpha}: E_{\alpha} \rightarrow S^{n}$ the principal $S^{3}$-bundle classified by $\alpha_{0}: S^{n} \rightarrow B S^{3}$. They show that there is a supply of $\alpha, \beta \in \pi_{n-1}\left(S^{3}\right)$ with $\alpha \neq \pm \beta$, which guarantees that $E_{\alpha} \not E_{\beta}$, and with $p_{\alpha} \circ \beta_{0} \simeq 0$ $\simeq p_{\beta} \circ \alpha_{0}$, which implies that the fibered product $E_{\alpha \beta}$ of the maps $p_{\alpha}$ and $p_{\beta}$ satisfies $E_{\alpha} \times S^{3} \simeq E_{\alpha \beta} \simeq E_{\beta} \times S^{3}$. The resulting homotopy equivalence $E_{\alpha} \times S^{3} \rightarrow E_{\beta} \times S^{3}$, which is not made explicit in [3], cannot be of the form $f \times g$ for then $f: E_{\alpha} \rightarrow E_{\beta}$ would induce isomorphisms on homotopy and hence would be a homotopy equivalence; it must be twisted. We present here these examples from the point of view of the cellular structure of the spaces $E_{\alpha} \times S^{3}$ and $E_{\beta} \times S^{3}$ to indicate how the homotopy equivalence $E_{\alpha} \times S^{3} \rightarrow E_{\beta} \times S^{3}$ can be generated by a twisted homotopy equivalence $S^{3} \times S^{3} \rightarrow S^{3} \times S^{3}$.

Let $m_{r}: S^{3} \times S^{3} \rightarrow S^{3}(r=0,1, \cdots, 11)$ be the twelve multiplications on $S^{3}$ as enumerated by M. Arkowitz and C. R. Curjel in [1]. For $\alpha: S^{n-1} \rightarrow S^{3}$ define the map

$$
g_{\alpha, r}=\alpha \times 1 \circ m_{r}: S^{n-1} \times S^{3} \rightarrow S^{3} \times S^{3} \rightarrow S^{3}
$$

(observing the "Hilton-Wylie" convention of writing composition of maps) and the adjunction space $E_{\alpha, r}=S^{3} \cup_{o_{\alpha, r}} B^{n} \times S^{3}$. These spaces are related to the principal $S^{3}$-bundles in that $E_{\alpha, 0}=E_{\alpha}[3$, Proposition 2.1]. We prove in $\S 3$ the following result.

THEOREM 1. Let $\alpha, \beta: S^{n-1} \rightarrow S^{3}$ be used to construct $E_{\alpha, r}$ and $E_{\beta, 8}$. If there exist integers $n_{i j}(i, j=1,2)$ such that

(i) $\operatorname{det}\left(n_{i j}\right)= \pm 1$,

(ii) $n_{11} \alpha \simeq \beta$ and $n_{12} \alpha \simeq 0$,

Received by the editors February 4, 1970.

AMS 1969 subject classifications. Primary 5540.

Key words and phrases. Cancellation for products, finite CW complexes, twisted homotopy equivalence. 
(iii) $n_{1 j}^{2}(2 s+1) \equiv n_{1 j}(2 r+1) \bmod 24(j=1,2)$, and if,

(iv) either $s=0,2,3,5,6,8,9$, or 11 ,

then there is a twisted homotopy equivalence $\left\{\left(\bar{n}_{i j}\right)\right\}: S^{3} \times S^{3} \rightarrow S^{3} \times S^{3}$ which extends to a homotopy equivalence $E_{\alpha, r} \times S^{3} \rightarrow E_{\beta, s} \times S^{3}$.

For the remainder of this section we restrict our attention to $r=s$ from the list (iv). Using Theorem 1 we can reprove [3, Corollary 2.2] and [3, Theorem 2.5].

THEOREM 2. Let $\alpha$ be of order $k, k_{0}=\operatorname{gcd}(k, 24), \iota$ prime to $k, \iota \equiv 1$ $\bmod k_{0}, \beta=\iota \sigma$. Then $E_{\alpha, r} \times S^{3} \simeq E_{\beta, r} \times S^{3}$.

PRoOF. Since $\iota \equiv 1 \bmod k_{0}$, we can find $n_{11} \equiv \iota \bmod k, n_{11} \equiv 1 \bmod 24$. Then $n_{11}$ is prime to $k$ and to 24 and hence to $n_{12}=24 k$. Thus we have integers $n_{i j}(i, j=1,2)$ with

(i) $\operatorname{det}\left(n_{i j}\right)=1$,

(ii) $n_{11} \alpha \simeq \iota \alpha=\beta$ and $n_{12} \alpha=0$, and

(iii) $n_{1 j}\left(n_{1 j}-1\right)(2 r+1) \equiv 0 \bmod 24(j=1,2)$.

As in [3, Theorem 2.3] we can see that $E_{\alpha, r} \simeq E_{\beta, s}$ implies that $\alpha \simeq \pm \beta$ and so we have the immediate consequence of Theorem 2 .

Theorem 3. Let $\alpha$ be of prime order $p \neq 2,3$, let $\iota$ be prime to $p$ $\iota \not \equiv \pm 1 \bmod p, \beta=\iota \alpha$. Then

$$
E_{\alpha, r} \times S^{3} \simeq E_{\beta, r} \times S^{3}, \quad E_{\alpha, r} \not E_{\beta, r}
$$

2. The abstract situation. We work in the category of $k$-spaces with base-point and base-point preserving maps, with composition of $f: A \rightarrow B$ and $g: B \rightarrow C$ written $f \circ g: A \rightarrow C$. The equivalence relation induced by homotopies which preserve base-points will be denoted by $\simeq$.

Given $g: X \times Y \rightarrow Z$ and the inclusion $c: X \rightarrow C X$ of $X$ onto the base of its cone $C X$, the adjunction space $Z \cup_{o} C X \times Y$ described by

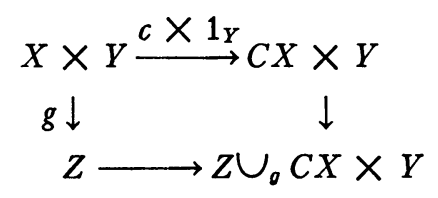

is Hausdorff and hence is a $k$-space $[4,2.6]$. We may therefore consider the above diagram as a push-out in the category of $k$-spaces. Since there is an unrestricted exponential law in this category, each product functor $-\times W$ preserves push-outs and hence 
Proposition 4. For any space $W$, the identity function

$$
1: Z \times W \cup_{o \times 1} C X \times Y \times W \rightarrow\left(Z \cup_{\diamond} C X \times Y\right) \times W
$$

is a homeomorphism.

Proposition 5. If $h: X \rightarrow X^{\prime}, k: Y \rightarrow Y^{\prime}$, and $v: Z \rightarrow Z^{\prime}$ are homotopy equivalences, and if $g: X \times Y \rightarrow Z$ and $g^{\prime}: X^{\prime} \times Y^{\prime} \rightarrow Z^{\prime}$ are maps such that $g \circ v \simeq h \times k \circ g^{\prime}: X \times Y \rightarrow Z^{\prime}$, then there is a homotopy equivalence

$$
Z \cup_{0} C X \times Y \rightarrow Z^{\prime} \cup_{0^{\prime}} C X^{\prime} \times Y^{\prime}
$$

extending $v: Z \rightarrow Z^{\prime}$.

Proof. When $Y$ and $Y^{\prime}$ are singletons, then $Z \cup_{g} C X \times Y$ and $Z^{\prime} \cup_{g^{\prime}} C X^{\prime} \times Y^{\prime}$ are the mapping cones of $g$ and $g^{\prime}$. In this special case the above result is standard and its proof $[2$, p. 40$]$ can easily be modified to cover the general case.

From now on let $Y$ and $Y^{\prime}$ be connected cellular spaces with multiplications $m: Y \times Y \rightarrow Y$ and $m^{\prime}: Y^{\prime} \times Y^{\prime} \rightarrow Y^{\prime}$ (i.e., the codiagonal maps $\nabla \simeq i \circ m: Y \vee Y \rightarrow Y \times Y \rightarrow Y$ and $\nabla \simeq i^{\prime} \circ m^{\prime}: Y^{\prime} \vee Y^{\prime} \rightarrow Y^{\prime} \times Y^{\prime}$ $\left.\rightarrow Y^{\prime}\right)$. Then given $\alpha: X \rightarrow Y$ and $\beta: X \rightarrow Y^{\prime}$ we form

$$
\begin{aligned}
& g_{\alpha}=\alpha \times 1 \circ \mathrm{m}: X \times Y \rightarrow Y \times Y \rightarrow Y, \\
& g_{\beta}=\beta \times 1 \circ m^{\prime}: X \times Y^{\prime} \rightarrow Y^{\prime} \times Y^{\prime} \rightarrow Y^{\prime},
\end{aligned}
$$

and the associated adjunction spaces $E_{\alpha}=Y \cup_{o_{\alpha}} C X \times Y$ and $E_{\beta}$ $=Y^{\prime} \cup_{o_{\beta}} C X \times Y^{\prime}$. From the previous two propositions we have

Corollary 6. (i) $E_{\alpha} \times Y=Y \times Y \cup_{\theta_{\alpha} \times 1} C X \times Y \times Y$ and $E_{\beta} \times Y^{\prime}$ $=Y^{\prime} \times Y^{\prime} \cup_{g_{\beta} \times 1} C X \times Y^{\prime} \times Y^{\prime}$.

(ii) If $k: Y \times Y \rightarrow Y^{\prime} \times Y^{\prime}$ is a homotopy equivalence for which

$$
\begin{aligned}
& X \times Y \times Y \stackrel{1 \times k}{\longrightarrow} X \times Y^{\prime} \times Y^{\prime} \\
& \begin{array}{ll}
\alpha \times 1 \times 1 & \downarrow \\
Y \times Y \times Y & Y^{\prime} \times Y^{\prime} \times Y^{\prime}
\end{array} \\
& \begin{array}{ccccc}
m \times 1 & \downarrow & & \downarrow & m^{\prime} \times 1 \\
& Y \times Y & \stackrel{k}{\rightarrow} & Y^{\prime} \times Y^{\prime} &
\end{array}
\end{aligned}
$$

is homotopy commutative, then $k$ extends to a homotopy equivalence $E_{\alpha} \times Y \rightarrow E_{\beta} \times Y^{\prime}$.

We now describe some special twisted homotopy equivalences $Y \times Y \rightarrow Y^{\prime} \times Y^{\prime}$. Let $Z$ be a space with multiplication $n: Z \times Z \rightarrow Z$. Given four maps $k_{i j}: W \rightarrow Z(i, j=1,2)$ we define $\left\{\left(k_{i j}\right)\right\}: W \times W \rightarrow Z$ 
$\times Z$ to be the map with projections $\left\{\left(k_{i j}\right)\right\} \circ p_{j}=p_{1} \circ k_{1 j}+_{z} p_{2} \circ k_{2 j}$ : $W \times W \rightarrow Z(j=1,2)$, where the $\operatorname{sum} f{ }{ }_{z} g: A \rightarrow Z$ means $\Delta \circ f \times g \circ n:$ $A \rightarrow Z$. For example, if $\delta_{i j}: Y \rightarrow Y$ is given by $0,1_{Y}$ as $i \neq j, i=j$, then $\left\{\left(\delta_{i j}\right)\right\} \simeq 1: Y \times Y \rightarrow Y \times Y$. We can consider the four maps $k_{i j}: W \rightarrow Z$ $(i, j=1,2)$ as determining a $2 \times 2$ matrix $\left(k_{i j}\right)$. We write $\left(k_{i j}\right) \simeq\left(h_{i j}\right)$ if $k_{i j} \simeq h_{i j}(i, j=1,2)$, and so $\left(k_{i j}\right) \simeq\left(h_{i j}\right)$ implies $\left\{\left(k_{i j}\right)\right\} \simeq\left\{\left(h_{i j}\right)\right\}: W$ $\times W \rightarrow Z \times Z$. For $k_{i j}: Y \rightarrow Y^{\prime}$ and $h_{i j}: Y^{\prime} \rightarrow Y(i, j=1,2)$ we define matrix multiplication

$$
\begin{aligned}
& \left(k_{i j}\right)\left(h_{i j}\right)=\left(\begin{array}{c}
k_{i 1} \circ h_{1 j} \underset{Y}{+} k_{i 2} \circ h_{2 j}
\end{array}\right), \\
& \left(h_{i j}\right)\left(k_{i j}\right)=\left(\begin{array}{c}
h_{i 1} \circ k_{1 j}+\underset{Y^{\prime}}{+} h_{i 2} \circ k_{2 j}
\end{array}\right),
\end{aligned}
$$

and we say $\left(h_{i j}\right)$ and $\left(k_{i j}\right)$ are inverses if these products $\left(k_{i j}\right)\left(h_{i j}\right) \simeq\left(\delta_{i j}\right)$ and $\left(h_{i j}\right)\left(k_{i j}\right) \simeq\left(\delta_{i j}^{\prime}\right)$. We do not claim that then $\left\{\left(k_{i j}\right)\right\} \circ\left\{\left(h_{i j}\right)\right\}$ $\simeq 1_{Y \times Y}$ and $\left\{\left(h_{i j}\right)\right\} \circ\left\{\left(k_{i j}\right)\right\} \simeq 1_{Y^{\prime} \times Y^{\prime}}$, but nevertheless we prove

Proposition 7. Given $k_{i j}: Y \rightarrow Y^{\prime}(i, j=1,2)$, the map $\left\{\left(k_{i j}\right)\right\}: Y$ $\times Y \rightarrow Y^{\prime} \times Y^{\prime}$ is a homotopy equivalence if the matrix $\left(k_{i j}\right)$ has an inverse.

Proof. For $g: S^{n} \rightarrow Y \times Y,(n \geqq 1)$,

$$
\begin{aligned}
g \circ\left\{\left(k_{i j}\right)\right\} \circ p_{j} & =g \circ\left(p_{1} \circ k_{1 j} \underset{Y^{\prime}}{+} p_{2} \circ k_{2 j}\right) \\
& =g \circ p_{1} \circ k_{1 j} \underset{Y^{\prime}}{+} g \circ p_{2} \circ k_{2 j} \\
& \simeq g \circ p_{1} \circ k_{1 j}+g \circ p_{2} \circ k_{2 j} \quad(j=1,2)
\end{aligned}
$$

where + is the homotopy associative-commutative binary operation determined by the standard comultiplication on $S^{n}$. If $\left(h_{i j}\right)$ is a matrix inverse for $\left(k_{i j}\right)$ then

$$
\left\{\left(k_{i j}\right)\right\} \text { of }\left\{\left(h_{i j}\right)\right\}_{f}=1: \pi_{n}(Y \times Y) \rightarrow \pi_{n}(Y \times Y)
$$

and

$$
\left\{\left(h_{i j}\right)\right\}_{\sharp} \circ\left\{\left(k_{i j}\right)\right\}_{\sharp}=1: \pi_{n}\left(Y^{\prime} \times Y^{\prime}\right) \rightarrow \pi_{n}\left(Y^{\prime} \times Y^{\prime}\right) .
$$

For example,

$$
g \circ\left\{\left(k_{i j}\right)\right\} \circ\left\{\left(h_{i j}\right)\right\} \simeq g \quad \text { for } g: S^{n} \rightarrow Y \times Y \quad(n \geqq 1)
$$

since 


$$
\begin{aligned}
& g \circ\left\{\left(k_{i j}\right)\right\} \circ\left\{\left(h_{i j}\right)\right\} \circ p_{j}=g \circ\left\{\left(k_{i j}\right)\right\} \circ\left(p_{1} \circ h_{1 j}+\underset{Y}{+} p_{2} \circ h_{2 j}\right) \\
& =g \circ\left\{\left(k_{i j}\right)\right\} \circ p_{1} \circ h_{1 j} \underset{Y}{+} g \circ\left\{\left(k_{i j}\right)\right\} \circ p_{2} \circ h_{2 j} \\
& \simeq\left(g \circ p_{1} \circ k_{11}+g \circ p_{2} \circ k_{21}\right) \circ h_{1 j} \\
& +\left(g \circ p_{1} \circ k_{12}+g \circ p_{2} \circ k_{22}\right) \circ h_{2 j} \\
& =\left(g \circ p_{1} \circ k_{11} \circ h_{1 j}+g \circ p_{2} \circ k_{21} \circ h_{1 j}\right) \\
& +\left(g \circ p_{1} \circ k_{12} \circ h_{2 j}+g \circ p_{2} \circ k_{22} \circ h_{2 j}\right) \\
& \simeq\left(g \circ p_{1} \circ k_{11} \circ h_{1 j}+g \circ p_{1} \circ k_{12} \circ h_{2 j}\right) \\
& +\left(g \circ p_{2} \circ k_{21} \circ h_{1 j}+g \circ p_{2} \circ k_{22} \circ h_{2 j}\right) \\
& \simeq\left(g \circ p_{1} \circ k_{11} \circ h_{1 j}+g \circ p_{Y} \circ k_{12} \circ h_{2 j}\right) \\
& +\left(g \circ p_{2} \circ k_{21} \circ h_{1 j}+g \circ p_{2} \circ k_{22} \circ h_{2 j}\right) \\
& =g \circ p_{1} \circ\left(k_{11} \circ h_{1 j}+k_{12} \circ h_{2 j}\right) \\
& +\left(g \circ p_{2} \circ\left(k_{21} \circ h_{1 j}+\underset{Y}{k_{22} \circ h_{2 j}}\right)\right. \\
& \simeq g \circ\left\{\left(k_{i j}\right)\left(h_{i j}\right)\right\} \circ p_{j} \\
& \simeq g \circ\left\{\left(\delta_{i j}\right)\right\} \circ p_{j} \simeq g \circ p_{j}
\end{aligned}
$$

for $j=1,2$.

Thus $\left\{\left(k_{i j}\right)\right\}: Y \times Y \rightarrow Y^{\prime} \times Y^{\prime}$, as a weak homotopy equivalence between connected cellular spaces, is a homotopy equivalence.

TheOREM 8. Let $\alpha: X \rightarrow Y$ and $\beta: X \rightarrow Y^{\prime}$ be used to construct $E_{\alpha}$ and $E_{\beta}$ as prior to Corollary 6. If there exist four maps $k_{i j}: Y \rightarrow Y^{\prime}(i, j=1,2)$ such that

(i) the matrix $\left(k_{i j}\right)$ is invertible,

(ii) $\alpha \circ k_{11} \simeq \beta: X \rightarrow Y^{\prime}$ and $\alpha \circ k_{12} \simeq 0: X \rightarrow Y^{\prime}$,

(iii) $k_{1 j}: Y \rightarrow Y^{\prime}$ is an $H$-map for $j=1,2$, and if

(iv) the multiplication $m^{\prime}: Y^{\prime} \times Y^{\prime} \rightarrow Y^{\prime}$ is homotopy associative, then the map $\left\{\left(k_{i j}\right)\right\}: Y \times Y \rightarrow Y^{\prime} \times Y^{\prime}$ is a homotopy equivalence which extends to a homotopy equivalence $E_{\alpha} \times Y \rightarrow E_{\beta} \times Y^{\prime}$

Proof. Condition (i) and the previous proposition show that $\left\{\left(k_{i j}\right)\right\}$ is a homotopy equivalence. We use conditions (ii), (iii), and (iv) to show that

$\alpha \times 1 \times 1 \circ m \times 1 \circ\left\{\left(k_{i j}\right)\right\} \simeq 1 \times\left\{\left(k_{i j}\right)\right\} \circ \beta \times 1 \times 1 \circ m^{\prime} \times 1$, 
so that Corollary 6 is applicable:

$\alpha \times 1 \times 1 \circ m \times 1 \circ\left\{\left(k_{i j}\right)\right\} \circ p_{j}$

$$
\begin{aligned}
& \simeq \alpha \times 1 \times 1 \circ m \times 1 \circ\left(p_{1} \circ k_{1 j} \underset{Y^{\prime}}{+} p_{2} \circ k_{2 j}\right) \\
& \simeq \alpha \times 1 \times 1 \circ m \times 1 \circ k_{1 j} \times k_{2 j} \circ m^{\prime} \\
& \simeq \alpha \times 1 \times 1 \circ k_{1 j} \times k_{1 j} \times k_{2 j} \circ m^{\prime} \times 1 \circ m^{\prime} \\
& \simeq\left(\alpha \circ k_{1 j}\right) \times k_{1 j} \times k_{2 j} \circ 1 \times m^{\prime} \circ m^{\prime} \quad(j=1,2)
\end{aligned}
$$

while

$$
1 \times\left\{\left(k_{i j}\right)\right\} \circ \beta \times 1 \times 1 \circ m^{\prime} \times 1 \circ p_{1}
$$

$$
\begin{aligned}
& =1 \times\left(\left\{\left(k_{i j}\right)\right\} \circ p_{1}\right) \circ \beta \times 1 \circ m^{\prime} \\
& \simeq 1 \times\left(k_{11} \times k_{21} \circ m^{\prime}\right) \circ \beta \times 1 \circ m^{\prime} \\
& =1 \times k_{11} \times k_{21} \circ 1 \times m^{\prime} \circ \beta \times 1 \circ m^{\prime} \\
& =\beta \times k_{11} \times k_{21} \circ 1 \times m^{\prime} \circ m^{\prime} \\
& \simeq\left(\alpha \circ k_{11}\right) \times k_{11} \times k_{21} \circ 1 \times m^{\prime} \circ m^{\prime}
\end{aligned}
$$

and

$1 \times\left\{\left(k_{i j}\right)\right\} \circ \beta \times 1 \times 1 \circ m^{\prime} \times 1 \circ p_{2}=p_{Y \times Y} \circ\left\{\left(k_{i j}\right)\right\} \circ p_{2}$

$$
\begin{aligned}
& \simeq p_{Y \times Y} \circ k_{12} \times k_{22} \circ m^{\prime} \\
& \simeq 0 \times k_{12} \times k_{22} \circ 1 \times m^{\prime} \circ m^{\prime} \\
& \simeq\left(\alpha \circ k_{12}\right) \times k_{12} \times k_{22} \circ 1 \times m^{\prime} \circ m^{\prime},
\end{aligned}
$$

where $p_{Y \times Y}: X \times Y \times Y \rightarrow Y \times Y$ is projection on the last two factors.

3. A concrete case. Let $X=S^{n-1}$, let $Y$ be the three sphere $S^{3}$ with multiplication $m_{r}$, and let $Y^{\prime}$ be the three sphere $S^{3}$ with multiplication $m_{s}$. We claim that Theorem 1 is merely a rewording of Theorem 8 and we present the following facts in justification:

(i) For each integer $n$, let $[n]: S^{3} \rightarrow S^{3}$ be a map of degree $n$. Since $[n+m] \simeq[n]+[m]$, where $+=+_{Y},+_{Y^{\prime}}$, and $[n] \circ[m] \simeq[n m]$, then

$$
\begin{aligned}
\left(\left[n_{i j}\right]\right)\left(\left[m_{i j}\right]\right) & =\left(\left[n_{i 1}\right] \circ\left[m_{1 j}\right]+\left[n_{i 2}\right] \circ\left[m_{2 j}\right]\right) \\
& \simeq\left(\left[n_{i 1} m_{1 j}+n_{i 2} m_{2 j}\right]\right) .
\end{aligned}
$$

We conclude that the matrix of maps $\left(\left[n_{i j}\right]\right)$ is invertible iff the matrix of integers $\left(n_{i j}\right)$ is invertible, or equivalently, iff $\operatorname{det}\left(n_{i j}\right)= \pm 1$.

(ii) For $\alpha: S^{n-1} \rightarrow S^{3}$ and integer $n$, we have $\alpha \circ[n] \simeq n \alpha$. 
(iii) The map $[n]: S^{3}, m_{r} \rightarrow S^{3}, m_{s}$ is an $H$-map iff $n^{2}(2 s+1) \equiv$ $n(2 r+1) \bmod 24$ [1, Theorem A].

(iv) The multiplication $m_{8}: S^{3} \times S^{3} \rightarrow S^{3}$ is homotopy associative iff $s=0,2,3,5,6,8,9$, or 11 [1, Theorem B and Remark 1].

\section{REFERENCES}

1. M. Arkowitz and C. R. Curjel, Some properties of the exotic multiplications on the three-sphere, Quart. J. Math. Oxford Ser. (2) 20 (1969), 171-176.

2. P. Hilton, Homotopy theory and duality, Gordon and Breach, New York, 1965. MR 33 \#6624.

3. P. Hilton and J. Roitberg, On principal $S^{3}$-bundles over spheres, Ann. of Math. (2) 90 (1969), 91-107.

4. N. E. Steenrod, A convenient category of topological spaces, Michigan Math. J. 14 (1967), 133-152. MR 35 \#970.

The University of Oregon, Eugene, Oregon 97403 